\title{
Application de l'hypnose en médecine bucco-dentaire aux hôpitaux civils de Colmar
}

\section{Practice of the therapeutic hypnosis in the department of odontology of the civil hospitals of Colmar}

\section{Résumé}

\section{MOTS-CLES :}

- Hypnose, phobie, douleur dentaire.

\section{KEYWORDS: \\ - Hypnosis, phobia, dental pain.}

AOS 2013;262:10-12 DOI: $10.1051 / \mathrm{aos} / 2013203$ C) EDP Sciences 2013
Nous relatons l'expérience de la pratique de l'hypnose thérapeutique au sein du service d'odontologie des hôpitaux civils de Colmar. L'hypnose est largement pratiquée par notre assistante dentaire au service du patient phobique et sa mise en œuvre est décrite, avec un objectif celui de rassurer les patients anxieux et de les soulager pendant les soins.

\section{Abstract}

We report about the experimentation of the practice of the therapeutic hypnosis within the department of odontology of the civil hospitals of Colmar.

Hypnosis is widely practised by our dental assistant on the phobic patient and its implementation is described, with one objective: reassuring and relieving the anxious patients during the care.

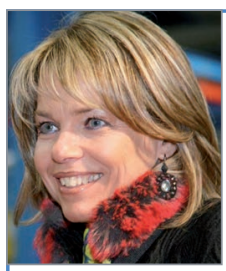

- Anne ROMBOURG, Praticien hospitalier,

Chef du service d'odontologie des hôpitaux civils de Colmar, 39, avenue de la Liberté, 68024 Colmar.

Karine BARMAS, Assistante dentaire au service d'odontologie des hôpitaux civils de Colmar, 39, avenue de la Liberté, 68024 Colmar.

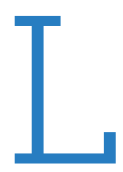

e contexte du cabinet dentaire alimente chez beaucoup de patients un état centré sur l'angoisse. Le recours à l'hypnose en médecine dentaire permet de remplacer cette peur par de la confiance.

En effet, l'hypnose peut être une aide efficace pour augmenter l'état de détente et le sentiment de sécurité. Si l'hypnose a de plus en plus d'adeptes chez les malades et les médecins, ce n'est pas par hasard ! On l'enseigne à la faculté de médecine depuis 2001, et l'Ordre des médecins l'a reconnue comme technique médicale en 2005.

\section{QU'EST-CE QUE L'HYPNOSE ?}

C'est un état de concentration et de relaxation qui permet à la part automatique et émotionnelle du comportement, l'inconscient, d'être plus réceptive qu'à l'état normal de « veille». C'est un état d'hypersuggestibilité, c'est-à-dire que l'on peut ressentir à volonté des émotions et des sensations imaginaires. On se sent bien, détendu.

On guide la personne jusqu'à un endroit où elle se sent en sécurité et on capte son attention grâce à des images ou à des couleurs pour qu'elle ne focalise pas sur les 


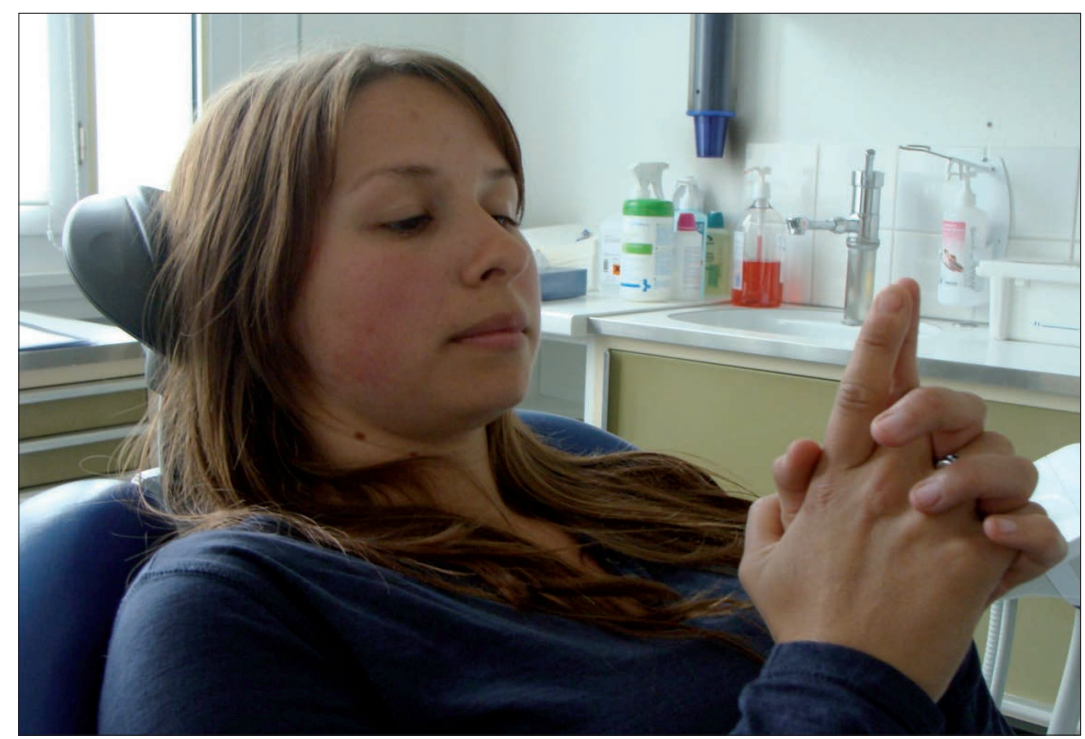

soins. Les résultats sont excellents, les patients ont besoin de moins d'anesthésiant, ils vivent mieux l'intervention et prennent moins d'antalgiques par la suite.

\section{LES CRITÈRES DE SÉLECTION}

Au sein de notre service, nous proposons le recours à l'hypnose pour :

$\checkmark$ le patient phobique, y compris l'enfant en âge de comprendre ;

\le patient au passé douloureux et aux séquelles posttraumatiques ;

$\checkmark$ le patient ayant des antécédents médicaux (cardiaques, crise de tétanie ou spasmophilie);

$\checkmark$ le patient présentant un vécu douloureux chez le dentiste ;

I ou tout simplement le patient demandeur.

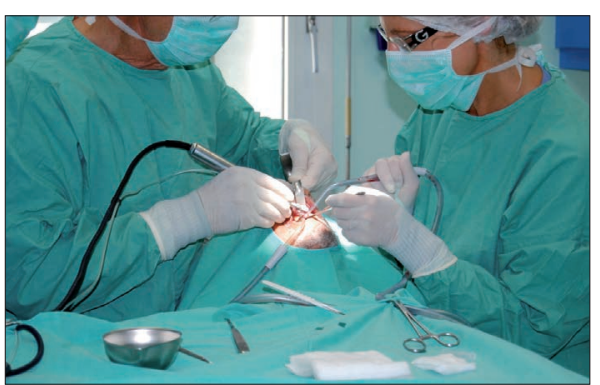

L'hypnose est réalisée pour tout type d'intervention, les soins, détartrage, avulsions dentaires simples, chirurgie bucco-dentaire (dents incluses...)

\section{COMMENT SE PASSE LA MISE EN CONDITION?}

L'hypnose sollicite la participation active du patient. Il s'agit d'un état de profonde relaxation, pendant lequel le patient va pouvoir s'exprimer librement. On peut d'ailleurs comprendre aisément l'efficacité de l'hypnose contre la douleur : des substances euphorisantes et anesthésiantes secrétées naturellement par l'hypothalamus permettent de mieux résister au stress, de soulager la douleur et de contrôler l'anxiété. Lors de l'état hypnotique, leur production est au maximum.

\section{L'hypnose conversationnelle}

Elle s'est avérée relativement aisée. Le plus simple est de se mettre au rythme du patient donc à son écoute et cela s'avère un outil puissant car le patient se retrouve au centre de notre préoccupation.

Un autre aspect puissant de cette hypnose conversationnelle est l'utilisation des métaphores, ce langage symbolique qui entraîne le patient vers un questionnement, un étonnement qui l'aide à se détacher du stress immédiat... On complète par de la musique douce et un rythme calme de l'équipe dentaire.

\section{L'hypnose formelle}

Sa mise en place nécessite un peu plus de temps au départ, car il faut dissiper les croyances concernant l'hypnose, définir un cadre avec le patient ainsi qu'un contrat, le but étant que le patient se sente libre, qu'il puisse " maîtriser " la séance et qu'il soit capable de décider ce qui est bon pour lui.

Nous proposons l'hypnose aux patients très anxieux, mais cela reste le choix du patient et non une obligation. Deux cas de figure se présentent :

$\checkmark$ le patient adulte ou enfant particulièrement anxieux, tant au niveau des soins que dans sa peur de perte de contrôle et qui refuse de se faire soigner : dans ce cas, on réalise une petite anamnèse et on consacre une séance d'hypnose avec induction d'une transe hypnotique et exploration d'un lieu agréable ou surtout, quand cela est possible, d'un lieu de sécurité sans réaliser de soins dentaires. Cela permet au patient de faire l'expérience de l'hypnose, de se rassurer avant de revenir dans un second temps pour les soins dentaires; 
le patient, adulte ou enfant anxieux qui a peur des soins mais qui, en temps normal, accepte de se laisser soigner.

Dans les deux cas, le patient se voit remettre un formulaire sur l'application de l'hypnose aux hôpitaux civils de Colmar.

Le jour de l'intervention, le patient se présente à la consultation, prend place en salle d'attente, pendant que l'assistante prépare le plateau technique en salle de soins. Elle invite le patient à s'installer confortablement dans le fauteuil dentaire. Puis elle fait une induction hypnotique idéo-motrice, (rapprochement des doigts, bras lourds etc., phénomène de persuasion). C'est après ces gestes que le patient est dans la position idéale pour commencer les soins et le praticien entre alors en jeu.

Il s'agit d'une parfaite synchronisation entre le praticien, l'assistante et le patient, l'hypnose à trois ! L'assistante annonce un exercice faisant fonctionner en même temps le corps et l'esprit, donne une convention de signaling :

tout d'abord détente, relaxation progressant sur le rythme respiratoire ;

validation de la réussite sans oublier de finir sur les mâchoires relâchées et fermeture des yeux ;

\suite en abordant la lèvre en regard de la dent à traiter, engourdie, anesthésiée ;

I valider la notion d'anesthésie ;

$\checkmark$ approfondissement de la transe ;

I suggestions post-hypnotiques qui permettent au patient d'en profiter au-delà de la séance.

\section{CONCLUSION}

L'hypnose, qu'elle soit conversationnelle ou formelle, apporte dans le cadre de l'activité d'un cabinet dentaire beaucoup de sérénité, une relation saine et agréable entre l'équipe dentaire et les patients et modifie de façon positive le comportement de chacun dans cette relation.

L'hypnose fonctionne à chaque fois que nous rejoignons le patient là où il se trouve et permet de réaliser les soins dans une ambiance beaucoup moins stressante. Elle facilite l'abord de ces soins que les patients redoutent et qu'ils reculent sans cesse.

D'autre part, des changements intérieurs interviennent chez le patient même s'ils n'ont pas été recherchés directement. Tout cela doit être réalisé dans le cadre de nos compétences, à l'intérieur d'un contrat établi avec le patient, et des objectifs clairs pour chacun.

\section{Bibliographie}

[1] Malarewicz JA. Cours d'hypnose clinique. Etudes éricksonniennes. Issy-les-Moulineaux : ESF,
Coll. Sciences humaines appliqués, 1990.

[2] Erickson MH. L'hypnose thérapeutique. Issy-les-Moulineaux : ESF, Coll. Art de la Psychotherapie, 2012.

[3] Tenenbaum S. L'hypnose éricksonienne : un sommeil qui éveille. $3^{\mathrm{e}}$ éd..
Paris : InterEditions, Coll. Développement personnel et accompagnement, 2012.

[4] Revue Hypnose \& Thérapies brèves. Bruxelles : Editions Satas.

[5] Bellet P. L'hypnose. Paris : Editions Odile Jacob, 2002.

\section{$\bigcirc$}

